\title{
Balance Ability in Low Back Pain Patients With Lumbosacral Radiculopathy Evaluated With Tetrax: A Matched Case-Control Study
}

\author{
Kee Hoon Kim, MD, Min Jeong Leem, MD, Tae Im Yi, MD, Joo Sup Kim, MD, PhD, Seo Yeon Yoon, MD
}

Department of Rehabilitation Medicine, Bundang Jesaeng General Hospital, Seongnam, Korea

\begin{abstract}
Objective To compare postural balance ability in patients with low back pain between groups with and without lumbosacral radiculopathy.

Methods Patients who were referred for electromyography because of low back pain during the period from April 2017 through June 2018 were chosen as subjects. They were divided into groups with and without lumbosacral radiculopathy based on the results of electromyography. We used Tetrax (Sunlight Medical Ltd., Ramat Gan, Israel) to objectively evaluate postural balance ability, and to measure the fall risk, stability index, weight distribution index, and Fourier index.

Results Patients in the lumbosacral radiculopathy group showed significantly higher fall risk (73.25 vs. 38.00; $\mathrm{p}<0.05$ ), weight distribution index (8.57 vs. 5.00; $\mathrm{p}<0.05)$, and stability index (21.19 vs. 13.16; $\mathrm{p}<0.05)$ than those in the group without lumbosacral radiculopathy. The Fourier index at high-medium frequency was significantly increased in the lumbosacral radiculopathy group (8.27 vs. 5.56; $\mathrm{p}<0.05$ ), whereas weight-bearing on the side of radiculopathy was significantly decreased.

Conclusion Patients with lumbosacral radiculopathy have decreased postural balance compared with patients without this condition. Somatosensory disturbances in lumbosacral radiculopathy might cause postural balance impairment. Assessment and treatment plan not only for pain reduction but also for postural balance improvement should be considered in the management of patients with lumbosacral radiculopathy.
\end{abstract}

Keywords Radiculopathy, Postural balance, Back pain, Somatosensory disorders, Elcetomyography

\section{INTRODUCTION}

Low back pain (LBP) is one of the most frequently occurring musculoskeletal problems, and a lifetime prevalence of $>70 \%$ has been reported [1]. If the pain progresses, it could affect the functional status or qual-

Received July 29, 2019; Revised September 5, 2019; Accepted October 2, 2019; Published online May 29, 2020

Corresponding author: Seo Yeon Yoon

Department of Rehabilitation Medicine, Bundang Jesaeng General Hospital, 20, Seohyeon-ro 180 beon-gil, Bundang-gu, Seongnam 13497, Korea. Tel: +82-31-779-0627, Fax: +82-31-779-0635, E-mail: seoyeon0521@gmail.com

ORCID: Kee Hoon Kim (https://orcid.org/0000-0001-6060-5261); Min Jeong Leem (https://orcid.org/0000-0002-1296-5972); Tae Im Yi (https://orcid. org/0000-0001-6369-4677); Joo Sup Kim (https://orcid.org/0000-0001-7541-1603); Seo Yeon Yoon (https://orcid.org/0000-0002-0365-2923).

(c) This is an open-access article distributed under the terms of the Creative Commons Attribution Non-Commercial License (http://creativecommons.org/ licenses/by-nc/4.0) which permits unrestricted noncommercial use, distribution, and reproduction in any medium, provided the original work is properly cited. Copyright ( 2020 by Korean Academy of Rehabilitation Medicine 
ity of life, and previous studies have reported significant correlations among pain, disability, and quality of life in patients with LBP [2]. Radiculopathy, also known as low back-related leg pain or lumbosacral (LS) radicular syndrome, is one of the most common variations of LBP. Symptoms of radiculopathy are more persistent and severe than those of LBP, and radiculopathy has been reported to have a less favorable outcome and to consume more health resources [3].

The initial diagnosis of LS radiculopathy is based on patient history and physical examination findings. Electrophysiologic studies, such as needle electromyography (EMG), have long been considered the most useful methods for detecting radiculopathies, and are highly specific for root or nerve dysfunction $[4,5]$. Recently, with the development of medical imaging devices, magnetic resonance imaging (MRI) has been widely used to diagnose radiculopathy. However, EMG still has additional value in detecting ongoing denervation as a significant predictor of radiologic nerve root compression. A previous study has revealed the efficacy of EMG in patients with clinically suspected LS radiculopathy without nerve root involvement on MRI [5].

Postural balance control is an important skill required for successful walking and daily activities. Several organs with visual, auditory, vestibular, proprioceptive, positional, muscular, and cognitive functions are involved in postural balance control [6-8]. Reduced ability to control postural balance has been associated with ambulatory dysfunction and an increased risk of falls [9]. Balance impairments are frequently reported in stroke survivors, with $\mathbf{8 3 \%}$ having impaired balance after an acute stroke [10]. A recent study reported that patients with moderate to severe osteoarthritis had lower balance control ability than those with mild osteoarthritis [11]. Studies on postural balance ability in patients with LBP have revealed decreased postural balance in these patients [12-15]. There have been some studies on postural balance ability in patients with LS radiculopathy, and most of them diagnosed radiculopathy based only on radicular symptoms [16-18]. Thus, we included patients with LS radiculopathy diagnosed using EMG.

Several methods are available for evaluating balance ability, such as the functional reach test or Berg Balance Scale test. These methods have been reported to have a high intra-class correlation and high reliability between test and retest [19]; however, these methods cannot evaluate balance in various aspects. Tetrax (Sunlight Medical Ltd., Ramat Gan, Israel) is an objective device for evaluating postural balance ability that has been used in several previous studies [20,21], and has shown high test-retest reliability [22-24]. It can be used to examine visual, somatosensory, neurologic, and orthopedic conditions that can affect postural balance ability. Thus, in this study, we used Tetrax with measures of stability index (ST), weight distribution index (WDI), Fourier index (FI), synchronization index, and fall risk to determine the pathway involved in balance problems in patients with radiculopathy. The objective of this study was to compare postural balance ability in patients with LBP between groups with and without LS radiculopathy, by using a posturography system (Tetrax).

\section{MATERIALS AND METHODS}

\section{Subjects}

Patients who were referred for EMG examination for LBP during the period from April 2017 through June 2018 were chosen as subjects. Patients who were diagnosed with LS radiculopathy based on EMG results were selected as cases, and those who were not diagnosed with LS radiculopathy were included in the control group after matching for age, sex, weight, and height. Patients with diseases that could affect balance ability, such as vestibular apparatus problem, severe visual disturbances, peripheral neuropathy including diabetes mellitus, impaired cognition, and severe osteoarthritis, were excluded from the study. Vestibular apparatus and visual disturbance were checked through history taking. Patients were excluded if they had pain in their knee, ankle, or hip joint. Patients with any peripheral neuropathy detected on a nerve conduction study were also excluded. Demographic data including age, sex, height, weight, and body mass index (BMI) were recorded. The study was approved by Institutional Review Board of Bundang Jesaeng General Hospital, and the requirement for informed consent was waived (IRB No. RM17-13).

\section{EMG data}

EMG examination with a monopolar needle was performed in each patient by an experienced physiatrist. Multiple muscles were examined within the appropriate 
myotome, and adjacent myotomes (above and below) were examined based on clinical suspicion. Any denervation or reinnervation activity was noted. The presence of positive sharp waves or fibrillations in $\geq 2$ areas of sampling per muscle was considered proof of ongoing denervation [5]. Motor unit action potentials were assessed individually. The duration was compared with the normal values, and motor unit action potentials with increased duration were considered signs of reinnervation. Routine nerve conduction studies and late responses were used to exclude other conditions such as peripheral neuropathy or spinal cord injury [25].

\section{Balance assessment using Tetrax}

Balance ability was evaluated using Tetrax, which was equipped with two paired force plates for measuring vertical pressure fluctuations over both heels and feet. The ST, WDI, FI, synchronization index, and fall risk were measured using Tetrax. ST is a variable that shows the degree of postural sway to control and compensate for changes in posture. The total amount of sway measured by the 4 force plates was totaled and divided by the patient's weight. The total amount of sway was calculated as the square root of the sum of the squared differences between adjacent pressure fluctuation signals, sampled at a rate of $32 \mathrm{~Hz}$. A higher ST indicates a more unstable posture [23]. The WDI reflects the level of weight distributed on the 4 force plates, with the normal index being 4 to 6 . The ideal posture is when $25 \%$ of the subject's weight is placed on each plate. An abnormally high WDI value is related to orthopedic and/or neurologic problems. Conversely, values close to zero are signs of excessive postural rigidity. FI is a regression parameter of the postural sway intensity analyzed using Fourier transform, which shows a different frequency for each lesion that causes instability. The Tetrax program compares the Fourier power values of posturographic performance to a mathematically computed regression curve, and evaluates the discrepancy between the graph obtained from the collected data and the theoretical "ideal" regression in the form of a coefficient. FI of low frequency (F1) is related to visual dysfunction. A high F1 signifies that the patient may have a visual problem [24]. The FI of the summation of low-medium frequencies (F2-4) suggests peripheral vestibular dysfunction, and the FI of the summation of high-medium frequencies (F5-6) is related to somato- sensory dysfunction $[25,26]$. Synchronization index is a score comparing 2 oscillation waves measured from the body vibrations of the 4 force plates. Synchronizations reflect the quality and efficiency of coordination movements of the agonist and antagonist muscle system of the lower extremities. The index ranges from $-1,000$ to 1,000 . It shows right and left weight-bearing ratios and foot and heel weight-bearing ratios. Fall risk is globally calculated using the computer system's data of the patient's ST, FI, WDI, and synchronization results. For all patients, the fall risk was calculated by considering the oscillation velocities computed by the posturographic software, and is expressed as a numerical value between 0 and 100 [23].

The normal eye open (NO) position was first evaluated followed by the normal eye closed (NC) position (to limit the effect of eyesight), thus emphasizing the effect of somatic sense or the vestibular organ. After placing rubber pillows on the floor of the machine, assessments with the patient standing on the pillows with the eyes open (PO position) and with the eyes closed (PC position) facing the front were conducted. The PO position limits only somatosensory function, whereas the PC position limits both visual and somatosensory functions (Fig. 1).

\section{Statistical analysis}

Data were analyzed using SPSS version 13.0 software (SPSS Inc., Chicago, IL, USA). The Kolmogorov-Smirnov

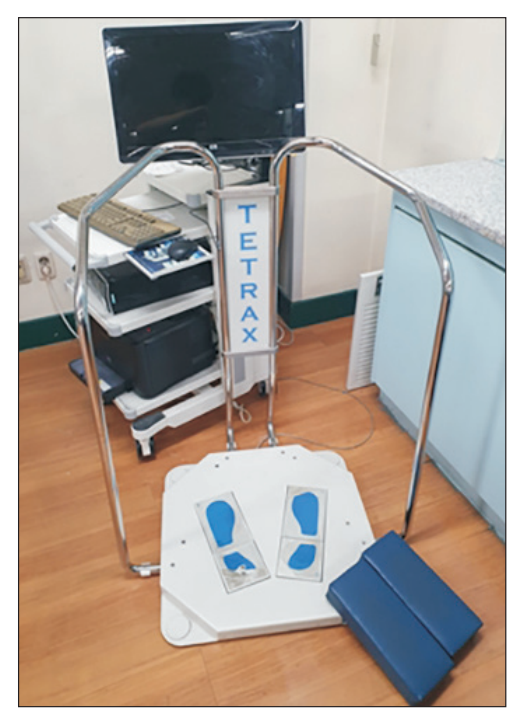

Fig. 1. Tetra-ataxiometric posturography (Tetrax; Sunlight Medical Ltd., Ramat Gan, Israel). 
test was used to test the normality of the variables. The chi-square and Mann-Whiney U-tests were used to evaluate categorical (sex and radiating pain) and continuous (age, height, weight, BMI, visual analogue scale score, and measures of balance ability) characteristics between the groups with and without LS radiculopathy. Wilcoxon signed-rank test was used to compare postural balance ability after closing the eyes or standing on pillows compared with the baseline status. If the p-value was $<0.05$, the result was considered statistically significant.

\section{RESULTS}

We recruited 20 patients with LS radiculopathy and 20 matched control patients without LS radiculopathy. The variables were not normally distributed $(p>0.05)$. The LS radiculopathy group consisted of 11 men and 9 women with a mean age of $56.36 \pm 13.30$ years, whereas the nonLS radiculopathy group consisted of 12 men and 8 women with a mean age of $56.23 \pm 15.82$ years. There was no significant difference between the two groups with respect to age or sex $(p>0.05)$. Moreover, there were no significant demographic differences with respect to weight, height, and BMI between the two groups. However, there was a significant difference in radiating pain and the visual analogue scale score, which was higher in the LS radiculopathy group (Table 1). LS spine MRI results were obtained in 15 patients in the LS radiculopathy group and in 11 patients in the non-LS radiculopathy group. The number of symptom-related MRI lesions was 13 in the LS radiculopathy group and 2 in the non-LS radiculopathy group. EMG showed that 3 patients had bilateral radiculopathy and the other 17 patients had unilateral radiculopathy. Of the 17 patients with unilateral radiculopathy, multilevel involvement was observed in 7 patients and only single-level involvement was seen in the other 10 patients (L4 level in 1 patient, L5 level in 6 patients, and S1 level in 3 patients).

The LS radiculopathy group showed significantly higher fall risk (73.25 vs. 38.00 ; $\mathrm{p}<0.05)$, WDI (8.57 vs. 5.00;

Table 1. Demographic and clinical data of patients

\begin{tabular}{|lccc}
\hline & With LS radiculopathy $(\mathbf{n = 2 0})$ & Without LS radiculopathy $(\mathbf{n}=\mathbf{2 0})$ & p-value \\
\hline Age $(\mathrm{yr})$ & $56.36 \pm 13.30$ & $56.23 \pm 15.82$ & 0.495 \\
\hline Sex & & & 0.343 \\
\hline Male & 11 & 12 & \\
Female & 9 & 8 & 0.344 \\
\hline Weight $(\mathrm{kg})$ & $69.52 \pm 9.72$ & $67.25 \pm 11.44$ & 0.871 \\
\hline Height $(\mathrm{cm})$ & $165.36 \pm 9.08$ & $164.14 \pm 9.59$ & 0.398 \\
\hline BMI $\left(\mathrm{kg} / \mathrm{m}^{2}\right)$ & $25.44 \pm 3.21$ & $24.85 \pm 3.03$ & $0.012^{*}$ \\
\hline VAS & $5.68 \pm 2.18$ & $3.95 \pm 2.74$ & $0.006^{*}$ \\
\hline Radiating pain & & & 10 \\
\hline Yes & 18 & 10 & \\
\hline No & 2 & & \\
\hline
\end{tabular}

Values are presented as mean \pm standard deviation.

LS, lumbosacral; BMI, body mass index; VAS, visual analog scale.

${ }^{*} \mathrm{p}<0.05$.

Table 2. Comparison of mean of fall risk, weight distribution index and stability index between the two groups

\begin{tabular}{lccc}
\hline & With LS radiculopathy $(\mathbf{n}=20)$ & Without LS radiculopathy $(\mathbf{n}=\mathbf{2 0})$ & p-value \\
\hline Fall risk & $73.25 \pm 24.50$ & $38.00 \pm 16.05$ & $0.000^{*}$ \\
Weight distribution index & $8.57 \pm 5.36$ & $5.00 \pm 2.30$ & $0.038^{*}$ \\
\hline Stability index & $21.19 \pm 7.02$ & $13.16 \pm 3.40$ & $0.010^{*}$ \\
\hline
\end{tabular}

Values are presented as mean \pm standard deviation.

LS, lumbosacral.

${ }^{*} \mathrm{p}<0.05$. 
$\mathrm{p}<0.05)$, and ST (21.19 vs. 13.16; $\mathrm{p}<0.05)$ than the control group (Table 2). The LS radiculopathy group showed a higher FI value at high-medium frequency ( 8.27 vs. 5.56; $\mathrm{p}<0.05)$ than the control group; however, there were no significant differences at low frequency (17.81 vs. 18.08; $\mathrm{p}>0.05)$ and low-medium frequency (27.49 vs. 22.07; p $>0.05$ ) (Fig. 2).

We additionally analyzed synchronization according to the side of the radiculopathy lesion. Seventeen patients were diagnosed with radiculopathy on only 1 side (8 patients on the right and 9 patients on the left).

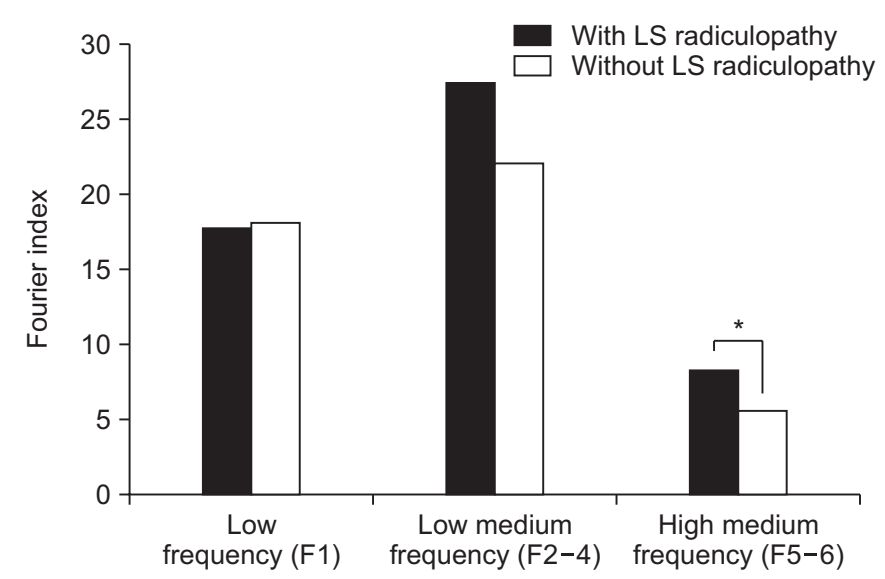

Fig. 2. Comparison of Fourier index between the two groups. LS, lumbosacral. * $\mathrm{p}<0.05$.
Weight-bearing on the side of the lesion was significantly reduced (synchronization index: 42.46 vs. 57.53; $\mathrm{p}<0.05$ ), and the same results were obtained in patients with rightsided radiculopathy (synchronization index: 40.32 vs. $59.67, \mathrm{p}<0.05)$ and those with left-sided radiculopathy (synchronization index: 44.36 vs. 55.63, $\mathrm{p}<0.05$ ). No significant difference in weight bearing was found between the forefoot and heel (Table 3).

With respect to the WDI, the LS radiculopathy group showed increased values $>8$ in each of the 4 positions, which indicates a weight distribution problem in patients with LS radiculopathy. In the assessments with pillows, the WDI was significantly increased in the non-LS radiculopathy group (PO position 7.82 vs. NO position 5.00, $\mathrm{p}<0.05$; PC position 6.50 vs. NC position $4.16, \mathrm{p}<0.05$ ). The LS radiculopathy group showed a significantly higher WDI than the control group in the NO and NC positions. However, in the PO and PC positions, no significant differences in WDI were observed between the two groups (Table 4).

\section{DISCUSSION}

In our study, patients with LS radiculopathy diagnosed using EMG showed decreased postural balance compared with those without LS radiculopathy. Weight bearing on the side of radiculopathy was significantly

Table 3. Comparison of synchronization index between lesion side and non-lesion side, fore foot and heel side

\begin{tabular}{lcccccc}
\hline & Lesion side & Non-lesion side & p-value & Fore foot & Heel & p-value \\
\hline Total patients $(\mathrm{n}=17)$ & $42.46 \pm 6.62$ & $57.53 \pm 6.62$ & $0.000^{*}$ & $50.48 \pm 3.03$ & $49.51 \pm 3.03$ & 0.653 \\
Rt. $\mathrm{rad}(\mathrm{n}=8)$ & $40.32 \pm 8.64$ & $59.67 \pm 8.64$ & $0.012^{*}$ & $50.83 \pm 3.83$ & $49.16 \pm 3.83$ & 0.674 \\
\hline Lt. $\mathrm{rad}(\mathrm{n}=9)$ & $44.36 \pm 2.84$ & $55.63 \pm 2.84$ & $0.008^{*}$ & $50.26 \pm 2.29$ & $49.82 \pm 2.29$ & 0.767 \\
\hline
\end{tabular}

Values are presented as mean \pm standard deviation.

Rt. rad, right lumbosacral radiculopathy; Lt. rad, left lumbosacral radiculopathy.

${ }^{*} \mathrm{p}<0.05$.

Table 4. Comparison of weight distribution index between normal position and position on pillows in two groups

\begin{tabular}{lcccccc}
\hline & NO & PO & p-value & NC & PC & p-value \\
\hline With LS radiculopathy $(\mathrm{n}=20)$ & $8.57 \pm 5.36$ & $8.07 \pm 4.32$ & 0.709 & $9.45 \pm 5.78$ & $8.17 \pm 4.43$ & 0.108 \\
Without LS radiculopathy $(\mathrm{n}=20)$ & $5.00 \pm 2.30$ & $7.82 \pm 3.43$ & $0.003^{*}$ & $4.16 \pm 2.35$ & $6.50 \pm 2.84$ & $0.002^{*}$ \\
p-value & $0.038^{*}$ & 0.925 & & $0.000^{*}$ & 0.265 &
\end{tabular}

Values are presented as mean \pm standard deviation.

LS radiculopahty, lumbosacral radiculopathy; NO, normal position with eyes open; PO, eyes open on pillows; NC, normal position with eyes closed; PC, eyes closed on pillows.

${ }^{*} \mathrm{p}<0.05$. 
decreased, and somatosensory function was disturbed in the group with LS radiculopathy.

The prevalence of radiculopathy from different studies ranged from $1.2 \%$ to $43 \%$ [3]. Radiculopathy has been suggested to provoke more severe pain than LBP, as well as to decrease the quality of life and to cause prolonged disability and absence from work [3,26-28]. Previous studies on radiculopathy mainly focused on pain reduction $[29,30]$. Medication, including both monotherapy and combination therapy, has shown efficacy in pain reduction, improving sleep disturbance, and alleviating anxiety $[28,31]$. Injections, such as caudal epidural steroid injection and transforaminal epidural steroid injection, have shown moderate to strong efficacy in managing lumbar root pain, and several other interventional techniques, including adhesiolysis and percutaneous disc compression, have demonstrated beneficial effects [32-34]. However, few studies have focused on postural balance problems in patients with LS radiculopathy.

Postural balance control is an important skill for gait and daily activities, and reduced ability to control postural balance has been associated with ambulatory dysfunction and an increased risk of falls [9]. Postural control represents a complex interplay between the sensory and motor systems, and involves perceiving environmental stimuli, responding to alterations in the body's orientation within the environment, and maintaining the body's center of gravity within the base of support [35]. Some studies on balance ability in patients with LBP have been published [12,14]. Mientjes and Frank [15] observed that patients with chronic LBP had increased sway compared with healthy people, and increased body sway was found when the subjects were tested with the eyes closed. Patients with LBP and radiculopathy demonstrated significant differences from control participants in terms of muscle activation timing, sequencing, and overall balance control [17]. Differences between the two groups were detected especially in the lower limb, and the authors suggested that radiculopathy may play a role in altering postural balance control [17]. In previous studies, radiculopathy was diagnosed using only the history of symptoms, such as pain, tingling sensation, or numbness in the legs. Whether the cause of decreased postural balance in patients with LBP and radiculopathy is of LBP origin or radiculopathy origin is unknown. Thus, we investigated postural balance ability in patients with radic- ulopathy diagnosed using EMG, and compared groups with and without LS radiculopathy to elucidate the effect of radiculopathy on postural balance ability.

Large and small sensory afferent nerve fibers are affected in LS radiculopathy [36]. Yamashita et al. [37] demonstrated that the functions of A-beta, A-delta, and C fibers are deteriorated in patients with LS radiculopathy. Somatosensory dysfunction has been associated with diminished motor performance [38], and our results were consistent with those of previous studies showing that the LS radiculopathy group had significantly increased highmedium frequency FI value, which implies that somatosensory disturbance affects postural balance in patients with radiculopathy. The WDI was higher than normal in the NO, PO, NC, and PC positions in the LS radiculopathy group, as the somatosensory function was already disturbed in these patients. In addition, there was no significant change after closing the eyes or standing on pillows in the LS radiculopathy group, probably because of the underlying somatosensory system pathology. Conversely, in the non-LS radiculopathy group, the WDI was in the normal range in the NO and NC positions, but was significantly increased above the normal range in the PO and PC positions. Standing on pillows caused somatosensory system disturbances, thus decreasing the postural balance ability. Assessment and treatment plans for not only pain reduction but also postural balance improvement should be considered in the management of patients with LS radiculopathy.

Our study had several limitations. The first limitation was the relatively small sample size. We included patients with LS radiculopathy only based on the results of EMG, to clarify the effect of radiculopathy on postural balance ability. Second, we did not perform other balance tests, such as the Berg Balance Scale test or functional reach test, and used Tetrax to assess postural balance. Because we attempted to elucidate the pathophysiology of decreased postural balance in patients with LS radiculopathy, we used Tetrax, which can evaluate various organ systems related to postural balance ability. Further studies on the effects of balance training in patients with LS radiculopathy would be needed.

In conclusion, we compared postural balance ability in patients with LBP between groups with and without LS radiculopathy diagnosed using EMG. The LS radiculopathy group showed decreased postural balance compared 
with the non-LS radiculopathy group. Somatosensory function was disturbed in the LS radiculopathy group, and weight bearing on the side of radiculopathy was significantly decreased. Postural balance ability should be evaluated in patients with LS radiculopathy to prevent fall injury, and an exercise program focusing on proprioception and postural balance ability would be needed in the management of these patients.

\section{CONFLICT OF INTEREST}

No potential conflict of interest relevant to this article was reported.

\section{AUTHOR CONTRIBUTION}

Conceptualization: Yoon SY. Methodology: Kim KH, Yoon SY. Formal analysis: Kim KH, Yoon SY. Project administration: Kim KH, Leem MJ, Yi TI, Kim JS, Yoon SY. Visualization: Kim KH, Yoon SY. Writing - original draft: Kim KH, Yoon SY. Writing - review and editing: Kim KH, Leem MJ, Yi TI, Kim JS, Yoon SY. Approval of the final manuscript: all authors.

\section{REFERENCES}

1. Andersson GB. Epidemiological features of chronic low-back pain. Lancet. 1999;354:581-5.

2. Kovacs FM, Abraira V, Zamora J, Teresa Gil del Real M, Llobera J, Fernandez C, et al. Correlation between pain, disability, and quality of life in patients with common low back pain. Spine (Phila Pa 1976) 2004;29:206-10.

3. Konstantinou K, Dunn KM. Sciatica: review of epidemiological studies and prevalence estimates. Spine (Phila Pa 1976) 2008;33:2464-72.

4. Aminoff MJ, Goodin DS, Parry GJ, Barbaro NM, Weinstein PR, Rosenblum ML. Electrophysiologic evaluation of lumbosacral radiculopathies: electromyography, late responses, and somatosensory evoked potentials. Neurology 1985;35:1514-8.

5. Coster S, de Bruijn SF, Tavy DL. Diagnostic value of history, physical examination and needle electromyography in diagnosing lumbosacral radiculopathy. J Neurol 2010;257:332-7.

6. Prieto TE, Myklebust JB, Hoffmann RG, Lovett EG,
Myklebust BM. Measures of postural steadiness: differences between healthy young and elderly adults. IEEE Trans Biomed Eng 1996;43:956-66.

7. Brandt T, Dieterich M. Postural imbalance in peripheral and central vestibular disorders. In: Bronstein AM, Brandt T, Woollacott MH, Nutt JG, editors. Clinical disorders of balance, posture and gait. 2nd ed. London: Arnold; 2004. p. 146-62.

8. Schieppati M, Nardone A. Free and supported stance in Parkinson's disease: the effect of posture and 'postural set' on leg muscle responses to perturbation, and its relation to the severity of the disease. Brain 1991;114(Pt 3):1227-44.

9. Fernie GR, Gryfe CI, Holliday PJ, Llewellyn A. The relationship of postural sway in standing to the incidence of falls in geriatric subjects. Age Ageing 1982;11:11-6.

10. Tyson SF, Hanley M, Chillala J, Selley A, Tallis RC. Balance disability after stroke. Phys Ther 2006;86:30-8.

11. Kim HS, Yun DH, Yoo SD, Kim DH, Jeong YS, Yun JS, et al. Balance control and knee osteoarthritis severity. Ann Rehabil Med 2011;35:701-9.

12. Radebold A, Cholewicki J, Polzhofer GK, Greene HS. Impaired postural control of the lumbar spine is associated with delayed muscle response times in patients with chronic idiopathic low back pain. Spine (Phila $\mathrm{Pa}$ 1976) 2001;26:724-30.

13. Dvir Z, Daniel-Atrakci R, Mirovski Y. The effect of frontal loading on static and dynamic balance reactions in patients with chronic low back dysfunction. Basic Appl Myol 1997;7:91-6.

14. Alexander KM, LaPier TL. Differences in static balance and weight distribution between normal subjects and subjects with chronic unilateral low back pain. J Orthop Sports Phys Ther 1998;28:378-83.

15. Mientjes MI, Frank JS. Balance in chronic low back pain patients compared to healthy people under various conditions in upright standing. Clin Biomech (Bristol, Avon) 1999;14:710-6.

16. Frost LR, Bijman M, Strzalkowski ND, Bent LR, Brown $\mathrm{SH}$. Deficits in foot skin sensation are related to alterations in balance control in chronic low back patients experiencing clinical signs of lumbar nerve root impingement. Gait Posture 2015;41:923-8.

17. Frost LR, Brown SH. Muscle activation timing and balance response in chronic lower back pain patients 
with associated radiculopathy. Clin Biomech (Bristol, Avon) 2016;32:124-30.

18. Lin SI, Lin RM. Sensorimotor and balance function in older adults with lumbar nerve root compression. Clin Orthop Relat Res 2002;(394):146-53.

19. Lee JJ, Lee HJ, Park JH, Han EY, Kim MJ, Jung HY. The Korean version of Berg Balance Scale as an index of activity related to ambulation in subjects with stroke. J Korean Acad Rehabil Med 2007;31:400-3.

20. Zhang JG, Ishikawa-Takata K, Yamazaki H, Morita T, Ohta T. Postural stability and physical performance in social dancers. Gait Posture 2008;27:697-701.

21. Steinberg N, Eliakim A, Pantanowitz M, Kohen-Raz R, Zeev A, Nemet D. The effect of a weight management program on postural balance in obese children. Eur J Pediatr 2013;172:1619-26.

22. Akkaya N, Doganlar N, Celik E, Aysse SE, Akkaya S, Gungor HR, et al. TEST-retest reliability of TETRAX static posturography system in young adults with low physical activity level. Int J Sports Phys Ther 2015;10:893-900.

23. Zhang L, Weng C. Test-retest reliability of Tetrax posturographic balance assessment system in elderly. Chin J Rehabil Theory Pract 2011;17:637-9.

24. Christensen IK, Deilami SS, Amiri S, Nissen MH, Devantier L, Ovesen T. Validation of posturographic measurements in adolescents. Otol Neurotol 2018;39:e568-e574.

25. Nardin RA, Patel MR, Gudas TF, Rutkove SB, Raynor EM. Electromyography and magnetic resonance imaging in the evaluation of radiculopathy. Muscle Nerve 1999;22:151-5.

26. Nykvist F, Hurme M, Alaranta H, Kaitsaari M. Severe sciatica: a 13-year follow-up of 342 patients. Eur Spine J 1995;4:335-8.

27. Selim AJ, Ren XS, Fincke G, Deyo RA, Rogers W, Miller $\mathrm{D}$, et al. The importance of radiating leg pain in assessing health outcomes among patients with low back pain. Results from the Veterans Health Study. Spine (Phila Pa 1976) 1998;23:470-4.

28. Boskovic K, Todorovic-Tomasevic S, Naumovic N, Grajic M, Knezevic A. The quality of life of lumbar radiculopathy patients under conservative treatment.
Vojnosanit Pregl 2009;66:807-12.

29. Bronfort G, Haas M, Evans RL, Bouter LM. Efficacy of spinal manipulation and mobilization for low back pain and neck pain: a systematic review and best evidence synthesis. Spine J 2004;4:335-56.

30. Rubinstein SM, van Middelkoop M, Assendelft WJ, de Boer MR, van Tulder MW. Spinal manipulative therapy for chronic low-back pain: an update of a Cochrane review. Spine (Phila Pa 1976) 2011;36:E825-46.

31. Saldana MT, Navarro A, Perez C, Masramon X, Rejas J. Patient-reported-outcomes in subjects with painful lumbar or cervical radiculopathy treated with pregabalin: evidence from medical practice in primary care settings. Rheumatol Int 2010;30:1005-15.

32. Boswell MV, Trescot AM, Datta S, Schultz DM, Hansen HC, Abdi S, et al. Interventional techniques: evidence-based practice guidelines in the management of chronic spinal pain. Pain Physician 2007;10:7-111.

33. Gibson JN, Grant IC, Waddell G. The Cochrane review of surgery for lumbar disc prolapse and degenerative lumbar spondylosis. Spine (Phila Pa 1976) 1999;24:1820-32.

34. Abdi S, Datta S, Trescot AM, Schultz DM, Adlaka R, Atluri SL, et al. Epidural steroids in the management of chronic spinal pain: a systematic review. Pain Physician 2007;10:185-212.

35. Shumway-Cook A, Woollacott MH. Motor control: theory and practical applications. 2nd ed. Philadelphia: Lippincott Williams \& Wilkins; 2001.

36. Nygaard OP, Mellgren SI. The function of sensory nerve fibers in lumbar radiculopathy: use of quantitative sensory testing in the exploration of different populations of nerve fibers and dermatomes. Spine (Phila Pa 1976) 1998;23:348-53.

37. Yamashita T, Kanaya K, Sekine M, Takebayashi T, Kawaguchi S, Katahira G. A quantitative analysis of sensory function in lumbar radiculopathy using current perception threshold testing. Spine (Phila $\mathrm{Pa}$ 1976) 2002;27:1567-70.

38. Priplata AA, Niemi JB, Harry JD, Lipsitz LA, Collins JJ. Vibrating insoles and balance control in elderly people. Lancet 2003;362:1123-4. 